The extent to which active movements are practised by the patient while the splint is still on, varies with different temperaments. Some cases require more encouragement than others. If after the removal of the splint there is still some limitation of movement at the metacarpo-phalangeal joints, the application of heat, preferably in the form of paraffin baths, followed by active movements, is found to be the most effective way of restoring mobility.

The position of fixation. Most cases can be treated with the hand in line with the forearm, as described above. In some cases, however, it is found that, although the reduction is easily performed, the deformity easily recurs. The correct position can in all cases be maintained if the wrist is flexed and is maintained flexed. This position is not adopted as a routine because the fingers cannot be so fully used and because it results in a delay in the return of movement in the wrist yet it must of necessity be sometimes adopted. No hard and fast rules can be laid down as to when to use the flexed position, but with experience it is possible to judge at the time of reduction whether the deformity is likely to recur, or whether the corrected position can be maintained by a plaster with the hand straight.

Failures in Treatment.

If an extremely critical attitude is adopted towards the results of Colles's fracture, it is found that in elderly patients a perfect wrist, indistinguishable from normal is not usually attained. There often remains a limitation of a few degrees of full flexion and extension of the wrist, which can only be detected on careful examination but of which the patient is unconscious. But if the mobility of the fingers is perfect, a quite considerable degree of limitation of movement at the wrist is no handicap. The purpose of these remarks is not to excuse such results, or to suggest any slackening in effort to obtain the perfect wrist, but to emphasize the supreme importance of encouraging the patient from the first day to perform full finger movements, and of seeing that they are performed. If the doctor is confident that finger movements can be performed, it is not difficult to communicate that confidence to the patient.

As in the treatment of all fractures, a detailed knowledge of the methods and technique is important: but it is even more important to grasp the general principles of fracture treatment, viz.:- - that successful functional end-results depend on reduction of deformity and on the maintenance of the correct position by the minimum fixation, which nevertheless must be adequate and combined with constant active movements of the whole of the rest of the limb.

\title{
THE DIAGNOSIS OF DISEASES OF THE URINARY TRACT IN GENERAL PRACTICE.*
}

By H. P. WINSBURY-WHITE, F.R.C.S. Surgeon, St. Paul's Hospital; Urologist, St. John's Hospital, Lewisham.

My object in choosing this subject for my lecture is largely to remind ourselves that when we suspect disease in the urinary tract, we will more quickly come to the right . onclusion, if the various steps of the examination of the patient are carried out in a certain definite order. I hasten to add that whether the patient complains

*A Post-Graduate Lecture delivered at St. Paul's Hospital, 18th October, 1933. 
of symptoms relating to the upper or the lower urinary tract, the method of examination should proceed in the same orderly way. I emphasize this point because one is so apt to rush straight to the kidney region because the symptoms relate to this part.

One fact which has impressed itself upon me by proceeding according to a certain routine, is how very common it is to find palpable evidence of a past genital infection in disease of the upper urinary tract. This applies to both sexes. I would like to make it quite clear before I proceed any further that the changes which occur in the upper urinary tract in these circumstances are not dependent upon the presence of some gross change in the lower urinary tract such as urethral stricture. Renal changes quite commonly occur long before such a stage is reached. I believe that many cases of renal stone and some cases of hydronephrosis arise in this way. Do you know that there is evidence of prostatitis in about 60 per cent. of renal calculus cases in men. When I talk about a past genital infection, I do not mean that the patient has necessarily had gonorrhoea, because I am convinced that a very large proportion of these cases are non-gonococcal in origin. My remarks apply just as much to females as to males. The difference being that the cervix replaces the prostate as a chronic focus of infection.

The following should be the routine method of procedure in the examination of a urological case. After the complaint is noted and the history taken, the first step is to inspect the external genitals. Here the presence of a phimosis possibly complicated by a chronic balanitis, or a small amount of turbid mucous visible at the external urinary meatus, perhaps only after squeezing the orifice, or some thickening of the epididymes, should always put one on one's guard that the urinary tract symptoms owe their origin most probably to some form of chronic infection, originating in the genitals. If the patient has passed his water just previously to the examination, then the immediate opportunity of detecting any urethral discharge has gone. One should therefore remember this in carrying out the examination. In determining whether the epididymes are normal, one should bear in mind that the tips of the thumb and forefinger should be able to meet along the posterior border of the testicle without the epididymis intervening. Thickening of the organ with adhesion to the testicle indicates a chronic inflammatory change. Chronic hydrocele must be regarded as equally significant and in my experience has been, almost without exception, associated with a gross chronic inflammatory change of the corresponding epididymis.

In turning to a rectal examination, the patient is conveniently placed in the kneeling position with the elbows on the couch, and the back straightened as much as possible. It is not uncommon to notice hæmorrhoids in cases of chronic genital infection and I believe them to be due to a backward spread of a thrombotic process from the pelvic floor in connection with inflammation of the internal genitals. On palpating the prostate, striking examples of simple or malignant enlargement, acute or chronic inflammation are easy enough to detect. The danger lies in assuming that the prostate is normal in the absence of any outstanding change which is obvious on rectal examination. Inflammatory debris can quite often be expressed from the prostate or seminal vesicles which give but the slightest or even no indication of disease, to the examining finger. In all cases therefore this opportunity should be taken of a gentle massage to these parts. The patient now gets down and is asked to pass all the water he can and to do it into two glasses, a couple of inches or so into the first and the rest into the second. The presence of inflammatory debris in the first and its absence from the second indicates some 
degree of inflammation of the internal genitals. Prostatic threads and pus in the first glass, with an equal or a greater quantity of pus in the second, means that the inflammation has spread from the prostate to the bladder.

The next step is to ask the patient to lie on the couch, and then to pass a catheter to ascertain first of all whether there is an appreciable obstruction and secondly whether or not there is any residual urine present. Unless one is dealing with an obvious case of retention bf urine, from simple prostatic enlargement, when a rubber catheter is the safest instrument, a selection of gum elastic coudé and blive headed catheters should be to hand. The French makes are better than the English chiefly because they are softer. As far as my experience goes, Eynard is the best French make. All the modern gum elastic catheters can be boiled for three or four minutes for quite a number of times before being badly damaged. I believe in commencing with a small calibre instrument, generally a No. 9 English scale (I6 Charriere). Remember that there is less likely to be an unpleasant reaction from passing a small catheter than from a large one. If an obstruction is encountered in the anterior urethra then one must resort to smaller sizes of instruments, as there is probably a stricture present. In this case one may fail completely to enter the bladder with a catheter, and, unless there is available a proper selection of soft-gum elastic filiform bougies, some of which should have cork screw tips, it would be better not to pursue these endeavours any further for the moment.

If one succeeds in getting a filiform through a difficult stricture, the instrument may be tied in along side of which the urine will slowly escape. In twelve hours time it should then be easy to pass a bigger instrument.

If a catheter succeeds in reaching the bladder, whether a stricture of theo anterior urethra is present or not, the residual urine must be kept and measured? If one fails to negotiate the stricture, the patient will almost certainly be able to pass his water, because such is the effect of the manipulation of the instrument against the stricture. In the meantime arrangements should be made for an anæsthetic to be given, under which an internal urethrotomy should be performed. I have seldom found it necessary to resort to supra-pubic cystotomy in order to relieve retention from a stricture.

To return to the question of residual urine, a most valuable piece of information is obtained by estimating this. I doubt if there is any more important part of the investigation than in carrying this out, for a sound opinion either on treatment or diagnosis is not possible if information on this point is lacking. At this stage it is as well to add that one of the late sequelæ of a chronic infection of the genitals, which almost invariably involves the neck of the bladder as well, is some stenosis of the internal urinary meatus. I would like to repeat that the condition is exceedingly common in my experience, more so than obstruction from a simple enlargement of the prostate, so that from the moment one realises that there is evidence of a chronic genital infection, the presence or absence of residual urine in the bladder is continually kept in mind.

Having carried out the investigation in the way I have indicated up to the point we have now reached, in a large number of urinary cases; the investigation is complete and the diagnosis is made. I wish to lay emphasis on this point because the class of case with urinary tract symptoms as a result of some disorder of the genital apparatus and neck of the bladder form a large proportion of the cases which a urologist is called upon to investigate. Other causes of residual urine are simple enlargement of the prostate and disease of the central nervous system. One 
should therefore not neglect to test the patient's reflexes. Even in those cases whose symptoms indicate stone in the bladder, or some upper urinary tract lesion, often all the trouble will be found to have started from the genital organs.

Now let us turn to the women. These seem to present a special problem all to themselves. Micturitional disturbance is so common in the female that such cases have almost come to be looked upon as a natural state with advancing years. This is of course quite the wrong attitude, as I can say at once that a proper examination of the patient will always enable one to identify the cause which as a rule is susceptible at least in some degree to treatment. But in order to reach the proper conclusions one must make a thorough examination of the extra-urinary pelvic organs. It is essential to approach these cases with the knowledge that one of the commonest sources of urinary tract disturbance is some type of abnormality of the reproductive organs, or pelvic floor. It is indeed the general lack of knowledge of this fact that has made these cases seem so puzzling and difficult to treat.

As a further example of this fact I would remind you that a coliform infection of the urinary tract is not unusual as a result of operative interference on any organ situated in or below the true pelvis. Where gross abnormalities are present these are soon identified and dealt with, but it is the minor ones which so regularly escape notice. I have in mind numerous cases of mild chronic inflammation involving the uterus and its adnexa, and the urethra.

In general practice I suppose it is not always convenient to examine the patient in the lithotomy position, but a serious attempt should be made to inspect the patient's vulvæ and cervix. If a special table is not available for this purpose, the lateral position, and a tubular vaginal speculum is the next best thing, not forgetting to have a good look at the external urinary meatus. In women it is much more easy to miss an acute urethritis or cervicitis than an infection in men, whether it be due to the gonococcus or a non-specific organism. This difficulty arises from the fact that the amount of discharge present is often insignificant, frequently not enough to attract the patient's attention. A mere enquiry as to the presence or absence of discharge is useless. . The fact of outstanding importance with regard to cystitis in women, is that in a very large proportion of cases, the infection originates in the urethra, or the cervix, and that nothing short of an inspection of the parts will suffice to determine whether they are free from blame. As far as the cervix is concerned any degree of erosion will explain a cystitis, and that with regard to the urethra, a chronic urethritis may be present with nothing to show for it on inspection. With the latter cases however, the diagnosis can usually be made by passing a catheter. A size I4 English rubber catheter should pass along a normal adult female urethra, without gripping, bleeding or pain, the presence of any of these features almost invariably means the presence of chronic urethritis. Sometimes a chronic vulvitis is to blame, in these circumstances urethritis and cystitis are both inevitable. There is no age when womanhood is exempt from the first apparently trivial but actually important condition.

In drawing off a specimen of urine by catheter from a female bladder, do not be deceived into thinking that inflammation can be ruled out as the cause of the patient's symptoms, because the urine appears perfectly clear on inspection, even when a laboratory report does not state the presence of pathological elements which indicate infection. It is a commonplace of cystoscopic examinations that a small area of mild inflammation is to be found in the vicinity of the internal urinary meatus in spite of the absence of corroborative evidence from the urine. The gratifying fact about identifying the urethra or the cervix as the origin of the 
patient's symptoms, is the readiness with which the earlier cases respond to treatment, and even the later ones can be helped to some extent. Intermittent urethral dilatation and dilatation and cauterisation of the cervix give the most gratifying results in the bulk of these cases.

While we are still talking of women, I would like to make some reference to that very common complaint, coliform infection of the urinary tract. It will save some time if I take this opportunity of saying that my remarks apply in many respects to men as well. I think I am right when I say that the notion is extremely prevalent in the profession that this complaint is a disease all by itself. This of course is wrong. It is but a phase of chronic urinary tract disease. Let us commence by recalling that the infection is found as a complication of almost every known pathological condition of the genital or the urinary apparatus. Its discovery should therefore be the signal for a thorough investigation. Incidentally chronic urethritis and cervicitis in women, and chronic urethritis and prostatitis and seminal vesiculitis in men, are the commonest initiating causes. In these circumstances the infection is first located in the lower urinary tract and later may ascend to the kidneys. But it is found complicating much more striking diseases than these, such as, hydronephrosis, renal calculus and genito-urinary tuberculosis, to mention only three.

I will refer very briefly to children. The common conditions that you will be called upon to deal with, are attacks of painful and frequent micturition, enuresis and pyelo-nephritis (pyelitis). With regard to the causes of these, in the male, be on the look-out for some atresia of the external urinary meatus, which gives rise to a coliform cystitis, and in the female be watchful for a vulvitis, this also results in a coliform cystitis. The initiating organism which is generally staphylococcus, or diphtheroid bacillus is soon replaced in an acute attack by the Bacillus coli. In the course of a year at the Princess Elizabeth of York Hospital for Children, I see a large number of cases which fall into one of these groups. Many of the pyelo-nephritis cases are found to have one of the genital infections mentioned, associated with it. The conditions are not only easy to recognise on inspecting the parts but respond so readily to treatment if the initial cause is tackled. In the males it is sometimes found that not only is the external urinary meatus unusually small, but that it is covered by a small brown crust. If this is gently removed the inflamed urethral mucosa is visible behind. In the female it is quite common to find an inflammatory condition of the vulvæ having its maximum on the vestibule which is the surface on to which the urethra opens. The orifice of the latter is invariably seen to be involved, hence the spread back to the bladder. It is perfectly easy as a rule to make a satisfactory examination of the parts without any resentment from the patient, by laying the child on its back, gently flexing the knees and then separating them widely. The labia majora are in this position readily drawn apart and the vulvæ fully exposed. This gives not only a view of the vulvæ, but of the posterior vaginal wall just inside the hymenal orifice. It is then usually noted that the inflammation does not involve the vagina at all. It is a vulvitis in its strictest sense.

A certain number of enuresis cases owe their symptoms to the conditions just specified but in the majority there are other causes, of which perhaps the most important is worms, so that a dramatic success may result when this unsuspected source of irritation is discovered and successfully treated. It is quite inadequate to be satisfied with an assurance from the mother that the child has no worms. I have frequently found them present, in spite of the parents belief to the contrary. My routine at the Children's Hospital at Shadwell is to admit all cases of enuresis 
for one week during which time they have three times daily, $\frac{1}{2}-I$ tablet Butolan, every other night on three occasions, $\frac{1}{2}-$ I grain of calomel, with a quassia enema the next morning. By the time this treatment is completed any existing intestinal parasites will be cleared away. Where there is found to exist a well-marked chronic urinary tract infection dilatation of some part of the urinary tract as a predisposing cause should always be thought of. The whole or any part of the apparatus may be involved. This question can only be settled, however, after special investigations have been made. Intravenous pyelography has proved to be of the greatest value in identifying these cases.

$\mathrm{A}^{\prime}$ few words of warning in a general way are not out of place. Many cases of renal colic have no stone present. Both renal infection and.hydronephrosis often give rise to this symptom. Do not expect that every case of hydronephrosis will present you with a swelling in the loin which can be felt. In the majority of cases no tumour can be palpated. Do not suppose that because a patient is not tender in one or other loin, that he does not suffer from renal tuberculosis. It is quite common for this physical sign to be absent in this type of case. Cystoscopy is often the quickest way of deciding whether urinary tuberculosis is present because of characteristic bladder changes, whereas bacteriological methods of search are unreliable except guinea-pig inoculation which takes six weeks. Do not neglect to order a cystoscopy in every case of hæmaturia. Always enquire from the patient whether the blood comes only at the end of micturition. This is the commonest type of bleeding and is due to cystitis as a rule. Exceptionally it is caused by a vesical calculus.

When we have to investigate symptoms relating to the upper urinary tract, we must always keep in mind that however sure we may feel that the patient has a stone or a hydronephrosis, we take upon ourselves an unjustifiable responsibility, by attempting to establish a final diagnosis without having recourse to radiography. The more recent refinements of this method of examination, make urinary tract diagnosis a very simple matter as a rule.

Cases of pain, colic and hæmaturia, all of renal origin, may still appear to be unexplained after a full investigation. One hastens to add that pyelography, especially by the intravenous method, will show changes characteristic of infection in the kidney in many of these patients when other means of investigation do not lead with such certainty to the same conclusion. Although it is for the specialist to make these finer interpretations of X-ray films, yet it is for the patient's medical attendant to know that the problem of elucidating symptoms can be solved in this way. $\mathrm{He}$ also has the knowledge that in certain difficult cases, cystoscopy is invaluable in order that diagnosis may be properly established.

In all chronic cases of arthritis, lumbago, sciatica, pains in the buttocks and thighs, never fail to make a careful examination of the genitals and lower urinary tract in the way I have indicated, which can be done quite easily in your consulting rooms. I think you will be surprised to find as a result of this, in how many cases the focus of infection is situated in one of these organs. The cervix in the female and the prostate in the male are the most common offenders.

And in conclusion one would go so far as to say that the means at our disposal of investigating the urinary tract seldom leave the correct diagnosis in doubt, if full use of these measures is made. On the other hand, investigations by special methods are often quite unnecessary, if the preliminary examination of the patient is carried out step by step in the manner I have indicated. 